\title{
Giant pulsations as modes of a transverse Alfvénic resonator on the plasmapause
}

\author{
P. N. Mager and D. Yu. Klimushkin \\ The Institute of Solar-Terrestrial Physics of Siberian Branch of Russian Academy of Sciences, Irkutsk, P.O.Box 291, 664033, Russia
}

(Received March 21, 2012; Revised October 1, 2012; Accepted October 2, 2012; Online published June 10, 2013)

\begin{abstract}
The paper assumes that the giant pulsations are oscillations trapped within a resonator resulting from finite plasma pressure on the outer edge of the plasmapause. This resonator is bounded, across the L-shells, by two turning points allowing the wave energy to be channeled azimuthally. This assumption can explain the basic properties of the giant pulsations: strong localization across magnetic shells, poloidal polarization, presence of a significant compressional component in the Pg magnetic field, the fact that their frequency does not depend on the radial coordinate. The wave field structure both across the L-shells and along the field lines is studied. In order to explain the amplitude modulation it is sufficient to suppose that the resonator is excited by some non-stationary process. Generation by a moving source comprised of substorm-injected particles is considered.
\end{abstract}

Key words: ULF waves, giant pulsations, poloidal modes, finite $\beta$, moving source, plasmapause.

\section{Introduction}

First identified by Kristian Birkeland in 1901, the giant pulsations $(\mathrm{Pg})$ were probably the first class of ULF waves identified in the terrestrial magnetosphere. They gained their name after Bruno Rolf, who exclaimed "True giant amongst dwarfs!" when studying one of those events (Rolf, 1931). Indeed, it had an amplitude of several tens of gammas, while most of the micropulsations found by then had dozens of times smaller amplitudes. Although later the giant pulsations lost their amplitude champion title, this term was assigned to waves similar to those observed by Birkeland and Rolf featuring predominant polarization in the $D$-component, long duration, quasi-sinusoidal shape with some amplitude modulation. Simulation of the Pg pulsations began with the papers by Kato and Watanabe (1955, 1956) and Lehnert (1956), who identified them with MHD waves in space plasmas. Now it is well established that Pgs represent a variety of guided poloidal Alfvén waves in the terrestrial magnetosphere, that is, waves with high azimuthal wave numbers, $m \gg 1$. Besides, the term "giant pulsations" is by no means improper: being high- $m$ waves, they are heavily attenuated by the atmosphere and must reach large enough amplitudes in space in order to be detected at the ground.

\subsection{Observable features of the giant pulsations: A syn- opsis}

Giant pulsations are usually observed in the Pc4-5 range, with amplitudes of several tens of $\mathrm{nT}$ at low geomagnetic activity (Ol', 1963; Annexstad and Wilson, 1968; Brekke et al., 1987; Taylor et al., 1989). They are characterized by a sinusoidal shape with some amplitude modulation, and by

Copyright (c) The Society of Geomagnetism and Earth, Planetary and Space Sciences (SGEPSS); The Seismological Society of Japan; The Volcanological Society of Japan; The Geodetic Society of Japan; The Japanese Society for Planetary Sciences; TERRAPUB.

doi: $10.5047 /$ eps.2012.10.002 a long duration of wave packets. The major part of their power is, according to data from ground magnetometers, in the $D$-component, which means that these waves are poloidally polarized in the magnetosphere (the field lines oscillate in the meridional direction). Spacecraft observations of Pgs often detect a considerable compressional magnetic field component as well (Hughes et al., 1979; Kokubun et al., 1989). They are usually registered in the auroral regions or just outside the plasmapause (Rostoker et al., 1979; Chisham et al., 1992; Takahashi et al., 2011), and sometimes within the plasmasphere (Green, 1985). Other kinds of poloidal Alfvén waves have been observed in the vicinity of the outer edge of the plasmapause (Takahashi and Anderson, 1992; Schäfer et al., 2008).

A necessary (although not sufficient: Klimushkin et al., 2004) condition for the Alfvén waves to be poloidally polarized is a high azimuthal wave number value, $|m| \gg 1$. Indeed, direct measurements confirm that Pgs are moderately high- $m$ waves, $|m|=15-40$. The azimuthal phase velocity is westward (negative $m$ values), that is, it coincides with the ion gradient-curvature drift direction. Some studies found that their localization region is only 600-700 $\mathrm{km}$ wide in the azimuthal (east-west) direction slowly drifting westward at a velocity typical of the 10-20 keV ion drift velocities (Glassmeier, 1980; Chisham et al., 1992). However, other studies failed to detect such a drift (Glassmeier et al., 1999).

Many studies also revealed that the Pgs are strongly localized across magnetic shells. Projected onto the Earth's surface, their localization region is only $200-300 \mathrm{~km}$ wide in the north-south direction, which corresponds to $\sim 1 R_{E}$ in the magnetosphere (e.g., Rostoker et al., 1979; Glassmeier, 1980; Chisham et al., 1992). The frequency does not change with the L-shell (Takahashi et al., 2011). As Chisham et al. (1997) found, the maximum of the $D$ component is observed on the same positions as the dip of 
the $H$-component. The latitudinal profile of $\mathrm{Pg}$ pulsations in the $D$-component can be described by a Gaussian function.

The parallel (field-aligned) structure of giant pulsations is a subject of major controversy. Comparing the observed Pgs' frequencies to those calculated in the quasi-dipole models relying on a power-law radial distribution of mass density, many authors concluded that Pgs represent the second ( $N=2$, or even) harmonic of the standing wave which has one node (Glassmeir, 1980; Poulter et al., 1983; Chisham and Orr, 1991; Chisham et al., 1992). On the other hand, observations at conjugate ground stations have detected the fundamental ( $N=1$, or odd) parallel harmonic (Green, 1979; Thompson and Kivelson, 2001). Satellite observations usually confirm the latter case (Kokubun et al., 1989; Takahashi et al., 1992, 2011; Glassmeier et al., 1999). We also prefer the latter interpretation because a simple power-law radial distribution of mass density does not adequately models the Alfvén speed variation across the plasmapause and the fact the observable Pgs are usually found just outside the plasmapause. It should be noted that Green (1985) observed three Pg events within the plasmapause and inferred the fundamental parallel harmonic by comparing the observed vs. calculated frequencies.

\subsection{Modeling and the purpose of this paper}

There is a general consensus that the high- $m$ waves are generated due to various kinetic instabilities in hot components of the magnetospheric plasma. Regarding Pgs, this opinion has some observational basis. Pgs usually have the same (westward) direction of the azimuthal phase velocity as the ion gradient-curvature drift. The Pgs localization region sometimes shows a westward drift at velocities of the same order as the 10-20 keV ion drift velocities. Moreover, Chisham et al. (1992) and Wright et al. (2001) found some association between several Pg events and substorminjected 10-20 keV particles. Another hint at the role of energetic particles in $\mathrm{Pg}$ generation is a close association between Pgs and Pc1 pearl pulsations (Kurazhkovskaya et al., 2004). Wave-particle interaction was also invoked in explaining Pg amplitude modulation (Pokhotelov et al., 2000a).

However, it is not clear which kind of kinetic instability may be responsible for their generation. Two kinds of instabilities are usually suggested (Southwood, 1976). The first is the bump-on-tail instability, an instability due to the nonmonotonic dependence of the ion distribution function on energy. Such a distribution can result from substorm injection, since faster protons reach a given point on the azimuthal coordinate earlier than the lower energy ones. Therefore high-energy particles are added to the local background plasma at a higher rate than low-energy particles (Glassmeier et al., 1999; Wright et al., 2001). The bumpon-tail distribution can also appear under steady state conditions due to the existence of a global magnetospheric electric field causing energy independent $\vec{E} \times \vec{B}$ drift (Chisham, 1996; Ozeke and Mann, 2001). Chisham (1996) suggested that the second mechanism may provide an explanation for the rarity of Pgs and their occurrence during geomagnetically quiet times. Several studies reported simultaneous observations of the bump-on-tail ion distribution functions and the poloidal pulsations very similar or identical to Pgs (Hughes et al., 1979; Baddeley et al., 2005).

The bump-on-tail instability is a natural candidate if Pgs represent the second parallel harmonic (Chisham and Orr, 1991; Chisham et al., 1992). Glassmeier et al. (1999) suggested that the bump-on-tail instability may also be a source of the fundamental harmonic Pg event, but they assumed the wave to be highly asymmetric due to different ionospheric conductivities at opposite magneto-conjugated points. This suggestion caused some controversy (Glassmeier, 2000; Mann and Chisham, 2000), so this point has not been finally established.

Another possible source of energy is an instability associated with an inward ion density gradient, which can occur in the ring current region. That suggestion was usually made in studies supposing Pgs to be the fundamental parallel harmonic of standing wave (Takahashi et al., 1992, 2011).

Uncertainty in our understanding of the Pgs parallel structure leads to difficulties when attempting to determine their generation mechanism. There are also some additional difficulties. As Mager and Klimushkin (2005) showed, the instability growth rate only weakly depends on the $m$ number. Therefore the instability cannot select a narrow range of the $m$ numbers, although the observed waves have welldefined $m$ values. Therefore, the instability can generate waves propagating in both azimuthal directions, thus even the direction of the azimuthal phase velocity cannot be explained (Mager and Klimushkin, 2005).

Furthermore, there are some theoretical difficulties with the instability. First, instabilities associated with the bumpon-tail distributions and plasma density gradients should not be considered as the only possible candidates for the Pg generation mechanism. Such factors as temperature gradients, pressure anisotropy can also generate the Alfvén waves (Pokhotelov et al., 1985; Klimushkin and Mager, 2011, 2012). Second, the entire instability theory has been established for the monochromatic waves (stationary wave field structure) only, although it is evident that wave generation is an impulsive process.

It is worth noting that the impulsive character of the wave generation assume that the wave is launched by a source switched on at some instant. The wave was absent before it was generated by the source, but it does not have to disappear with the termination of the source activity. If dissipation in the ionosphere-magnetosphere system is not too high, the wave generated by an impulsive source can last for quite a long time. In the case of Pgs, it can be two or more consecutive days (Rostoker et al., 1979).

A problem associated with the impulsive character of wave generation is a phase mixing phenomenon. At the initial onset of the perturbation all field lines oscillate with about the same phase and the wave field is characterized by a predominantly poloidal polarization. However, since each field line oscillates with its own eigenfrequency, the oscillations on neighboring magnetic shells rapidly acquire significant phase differences. As a consequence the wave acquires a very small spatial scale across magnetic field shells and, hence, becomes toroidally polarized to preserve the sourcefree nature of the magnetic field (e.g., Mann et al., 1997; Leonovich and Mazur, 1998; Klimushkin et al., 2012). In- 
clusion of instability into this picture only further complicates the problem. Even though the wave amplification rate decreases in the course of poloidal-to-toroidal transformation, it remains positive. Therefore the most amplified oscillations should be the toroidally polarized ones and the larger the instability, the larger the amplitude of the toroidal oscillation (Klimushkin, 2000; Klimushkin and Mager, 2004a; Klimushkin et al., 2007).

Although the phase mixing phenomenon is a firm output of the MHD theory, the expected change of the wave polarization has not been observed for the Pgs, which constitutes yet another peculiarity of these pulsations (Chisham et al., 1997). The only factor which can prevent the transformation is wave damping due to the finite ionospheric conductivity. If the wave does not have enough time for the transformation and remain poloidal in the course of all period or its existence, then the damping must be stronger than the instability. Thus, it is the ionospheric damping rather than the instability which is favorable to the poloidal polarization (Klimushkin, 2007). In this case, the role of the instability in the poloidal wave generation is not clear.

Based on earlier ideas of Zolotukhina (1974) and Guglielmi and Zolotukhina (1980), Mager and Klimushkin (2007, 2008) discussed another generation mechanism of high- $m$ waves. This involved emission by azimuthally drifting proton clouds injected during substorms, similar to the Cerenkov emission. Other analogies include the Kelvin ship waves and lee (mountain) waves in the atmosphere. This approach avoids some difficulties of the instability theory and has a firm observational basis (Zolotukhina et al., 2008; Mager et al., 2009a; Yeoman et al., 2010, 2012). Several cases were observed when the giant pulsations appeared at some azimuthal location at the same time as did a cloud of particles injected during substorm arrival at the same spot (Chisham et al., 1992; Wright et al., 2001).

Another peculiar feature of Pg pulsations, their narrow localization across magnetic shells, is usually postulated in theoretical studies but not explained (Chisham et al., 1997). The high localization across the L-shells is not unusual for the field line resonances (Tamao, 1965), but these low- $m$ resonances have toroidal rather than poloidal polarization (the $H$-component on the ground), while Pgs are poloidally polarized waves. Other kinds of poloidal waves also exhibit sharp localization across the L-shells (Singer et al., 1982; Engebretson et al., 1992; Takahashi and Anderson, 1992; Cramm et al., 2000; Schäfer et al., 2008). Besides, the maximum of the poloidal component in the field line resonance occurs at the same location as the maximum of the toroidal component, while for the giant pulsations the maximum of the poloidal component corresponds to the dip of the toroidal component (Chisham et al., 1997). A possible solution is suggested by the strong compressional magnetic field component of giant pulsations which is an indicator of the role of finite plasma pressure in ULF wave formation (Klimushkin et al., 2004). Indeed, Pgs are a subclass of the poloidal Alfvén wave, but the poloidal eigenfrequency is very sensitive to plasma pressure (e.g., Klimushkin, 1997; Mager and Klimushkin, 2002; Agapitov and Cheremnykh, 2011; Mazur et al., 2012). A combined effect of the field line curvature and finite plasma pressure can result in trans-

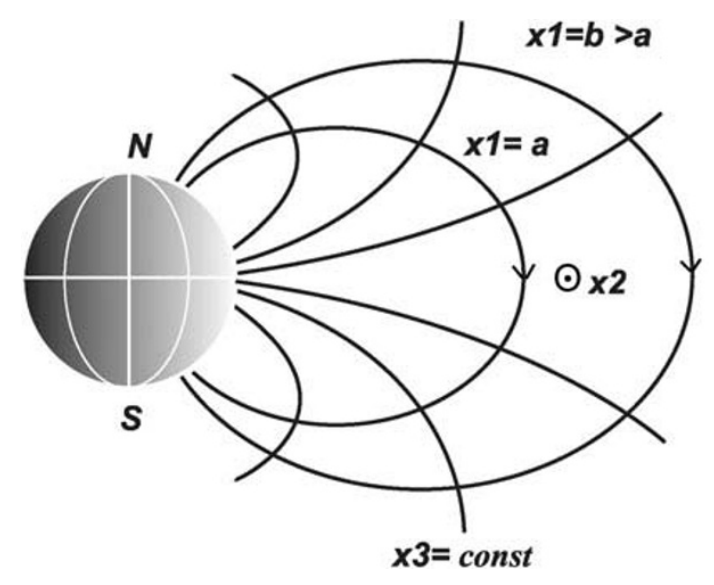

Fig. 1. Coordinate system.

verse resonators forming in some magnetospheric regions (plasmapause, ring current). The wave energy in these resonators is trapped between the conjugate ionospheres and cut-off shells, and is channeled along azimuth (Vetoulis and Chen, 1994; Denton and Vetoulis, 1998; Klimushkin, 1998). These resonator modes have a discrete spectrum determined by the field line curvature, plasma pressure, and the equilibrium current. The eigenfrequencies do not depend on the radial coordinate. Therefore, the resonator modes are not subject of the phase mixing phenomenon (Mager and Klimushkin, 2006).

Trapping of energy across magnetic shells may provide a natural explanation for the strong latitudinal localization of the poloidal modes. Under very general assumptions, the resonator can be situated on the outer edge of the plasmapause (Klimushkin et al., 2004), which may explain the location of the giant pulsations in that region. The purpose of this paper is to explore this suggestion, consider a possible generation mechanism of the wave in the resonator, and compare with observations.

\section{Main Equations}

Let us introduce a right-handed orthogonal curvilinear coordinate system $\left\{x^{1}, x^{2}, x^{3}\right\}$, in which the field lines are along the $x^{3}$ coordinate, i.e. lines along which the other two coordinates are invariable. The superscripts and subscripts denote the contravariant and covariant coordinates, respectively. In this coordinate system the stream lines are the $x^{2}$ coordinate lines, and the surfaces of constant pressure (magnetic shells) are the coordinate surfaces $x^{1}=$ const (Fig. 1). The $x^{1}$ and $x^{2}$ coordinates have the role of the radial and azimuthal coordinates, and will be represented using the McIlwain parameter $L$ and the azimuthal angle $\varphi$, respectively. It is convenient to choose a direction of the azimuthal coordinate coinciding with the proton drift direction. In order for the coordinate system to remain righthanded, the $x^{3}$ axis must be directed opposite to the ambient magnetic field. The physical length along a field line is expressed in terms of an increase of the corresponding coordinate as $d l_{3}=\sqrt{g_{3}} d x^{3}$, where $g_{3}$ is the component of the metric tensor, and $\sqrt{g_{3}}$ is the Lamé coefficient. Similarly, $d l_{1}=\sqrt{g_{1}} d x^{1}$, and $d l_{2}=\sqrt{g_{2}} d x^{2}$. The determinant of the metric tensor is $g=g_{1} g_{2} g_{3}$. 
Due to the infinite plasma conductivity, parallel electric field is absent, thus the wave's electric field lies on surfaces orthogonal to the field lines. An arbitrary vector field can be split into the sum of the potential and vortical components. By applying this theorem to a two-dimensional field $\vec{E}$, we put $\vec{E}=-\nabla_{\perp} \Phi+\nabla_{\perp} \times \vec{e}_{\|} \Psi$, where $\vec{e}_{\|}=\vec{B} / B$ and $\nabla_{\perp}$ is the transverse nabla-operator. The "potentials" $\Phi$ and $\Psi$ are associated with the electric field of the shear mode and the fast mode, respectively (Klimushkin, 1997). In the high- $m$ limit, the only ULF wave branch is the shear Alfvén mode, and the wave's electric field can be represented in the form

$$
\vec{E}=-\nabla_{\perp} \Phi
$$

Then, an inhomogeneous differential equation describing Alfvén oscillations is

$$
\mathcal{L}_{A} \Phi=Q\left(x^{1}, x^{2}, t\right)
$$

(Mager and Klimushkin, 2008). Here $Q\left(x^{1}, x^{2}, t\right)$ is the source term, and

$$
\begin{aligned}
\mathcal{L}_{A} & =\frac{\partial}{\partial x^{1}}\left[-\frac{\sqrt{g}}{g_{1}} \frac{1}{A^{2}} \frac{\partial^{2}}{\partial t^{2}}+\frac{\partial}{\partial x^{3}} \frac{g_{2}}{\sqrt{g}} \frac{\partial}{\partial x^{3}}\right] \frac{\partial}{\partial x^{1}} \\
& +\frac{\partial}{\partial x^{2}}\left[-\frac{\sqrt{g}}{g_{2}} \frac{1}{A^{2}} \frac{\partial^{2}}{\partial t^{2}}+\frac{\sqrt{g}}{g_{2}} \eta+\frac{\partial}{\partial x^{3}} \frac{g_{1}}{\sqrt{g}} \frac{\partial}{\partial x^{3}}\right] \frac{\partial}{\partial x^{2}}
\end{aligned}
$$

is the Alfvén differential operator. Here $A$ is the Alfvén speed and

$$
\eta=-2 K\left(\frac{4 \pi}{c} \frac{J}{B}+K \gamma \beta\right)
$$

where $K$ is the local field line curvature, $B$ and $J$ are the equilibrium magnetic field and the stationary current, $\gamma$ is the adiabatic index, and $c$ is the speed of light. Since the equilibrium current is related to the pressure $P$ gradient as $\nabla P=c^{-1} \vec{J} \times \vec{B}$, the $\eta$ parameter is different from zero only in finite pressure plasma (Denton and Vetoulis, 1998; Mager and Klimushkin, 2002; Klimushkin et al., 2004). On the order of magnitude,

$$
\eta \sim \frac{\beta}{L_{\perp}^{2}}
$$

where $L_{\perp}$ is the transverse plasma and magnetic field inhomogeneity scale roughly equal to the field line curvature radius.

The boundary conditions are chosen as

$$
\left.\Phi\right|_{x^{1}, x^{2} \rightarrow \pm \infty}=0,\left.\quad \Phi\right|_{x_{ \pm}^{3}}=0 .
$$

Here $x_{ \pm}^{3}$ denotes the intersection points of the field line and the ionosphere. The second condition corresponds to the wave fully reflected from the ionosphere.

For the high- $m$ ULF mode with frequency $\omega \ll k_{\perp} A$, where $k_{\perp}$ is the perpendicular component of the wave vector, the sum of the plasma and magnetic pressure perturbations is close to zero,

$$
\delta P+\frac{B \delta B_{\|}}{4 \pi}=0
$$

(e.g., Pokhotelov et al., 2000b), which presumes a nonzero compressional magnetic field component. It can be expressed through potential $\Phi$ as

$$
B_{\|}=\frac{c m}{\omega} \frac{1}{\sqrt{g_{1} g_{2}}} \frac{\eta}{2 K} \Phi
$$

(Klimushkin et al., 2004). Since according to $(3,4) \eta$ is a pressure dependent term, the observed compressional component of the poloidal ULF wave is evidence of the role of the plasma $\beta$ in wave formation. See also Klimushkin (1997) and Agapitov and Cheremnykh (2011).

In order to solve the wave equation (2), we Fourier transform this equation over $\varphi$ and $t$. The result is a differential equation with respect to two variables only, $x^{1}$ and $x^{3}$ :

$$
\hat{L}_{A} \Phi_{m \omega}=\tilde{q}_{m \omega}
$$

where $\omega$ and $m$ are the parameters of the Fourier transform over time (frequency) and azimuthal angle (azimuthal wave number), $\tilde{q}_{m \omega}$ is the Fourier-image of $Q(x, \varphi, t)$, and $\hat{L}_{A}$ is the Fourier-image of the Alfvénic operator $\mathcal{L}_{A}$ (that is, the Alfvénic operator for the monochromatic wave with frequency $\omega$ and azimuthal wave number $m$ )

$$
\hat{L}_{A} \equiv \frac{\partial}{\partial x^{1}} \hat{L}_{T}(\omega) \frac{\partial}{\partial x^{1}}-m^{2} \hat{L}_{P}(\omega),
$$

where

$$
\hat{L}_{T}(\omega)=\frac{\partial}{\partial x^{3}} \frac{g_{2}}{\sqrt{g}} \frac{\partial}{\partial x^{3}}+\frac{\sqrt{g}}{g_{1}} \frac{\omega^{2}}{A^{2}}
$$

is the toroidal mode operator, and

$$
\hat{L}_{P}(\omega)=\frac{\partial}{\partial x^{3}} \frac{g_{1}}{\sqrt{g}} \frac{\partial}{\partial x^{3}}+\frac{\sqrt{g}}{g_{2}}\left(\frac{\omega^{2}}{A^{2}}+\eta\right),
$$

is the poloidal mode operator. The eigenvalues of these operators with the boundary condition on the ionosphere (5) are denoted $\Omega_{T N}$ and $\Omega_{P N}$, respectively. They are called the toroidal and poloidal eigenfrequencies since they characterize the purely azimuthal (toroidal) and radial (poloidal) oscillations of the field lines. In a magnetosphere with curved field lines, the Alfvénic eigenfrequency depends on the direction of field line oscillations. The poloidal oscillations and the toroidal oscillations have different eigenfrequencies, as shown in Fig. 2 (e.g. Leonovich and Mazur, 1993). The $\eta$ term in Eq. (3) hints that the poloidal eigenfrequency is significantly influenced by the finite plasma pressure and the equilibrium current (Klimushkin, 1997; Mager and Klimushkin, 2002; Klimushkin et al., 2004; Agapitov and Cheremnykh, 2011; Mazur et al., 2012).

The method for solving Eq. (7) was developed in Leonovich and Mazur (1995) and Vetoulis and Chen (1994). It was shown there that the function $\Phi_{m \omega}$ can be represented as

$$
\Phi_{m \omega} \approx R_{N}\left(x^{1}\right) P_{N}\left(x^{1}, x^{3}\right)
$$

where $P_{N}\left(x^{1}, x^{3}\right)$ is an eigenfunction of the poloidal operator $\hat{L}_{P}$, defining the parallel structure of the $N$-th poloidal harmonic standing between the ionospheres. The normalization condition is

$$
\left\langle\frac{\sqrt{g}}{g_{2}} \frac{P_{N}^{2}}{A^{2}}\right\rangle=1
$$




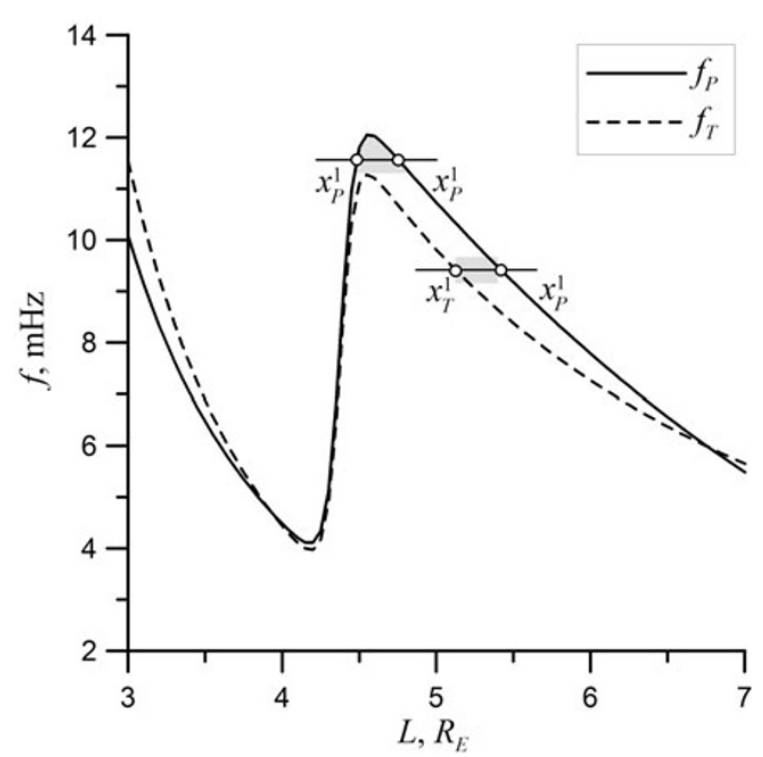

Fig. 2. Example of the behavior of the toroidal $f_{T}$ and poloidal $f_{P}$ eigenfrequencies (principal harmonics) with finite plasma pressure at medium geomagnetic activity. The filled areas are the transparent regions. The eigenfrequencies are calculated for the model plasma parameters in Schäfer et al. (2008).

where the angle brackets denote integration along the field line between the ionospheres, $\langle\cdots\rangle=\int_{x_{-}^{3}}^{x_{+}^{3}}(\cdots) d x^{3}$. The function $R_{N}\left(x^{1}\right)$ describes the structure of this harmonic across magnetic shells.

Further, if we substitute the function $\Phi_{m \omega}\left(x^{1}, x^{3}\right)$ from Eq. (8) into Eq. (7), we obtain an ordinary differential equation defining the radial structure of the wave field:

$$
\begin{aligned}
\frac{\partial}{\partial x^{1}}\left(\omega^{2}-\Omega_{T N}^{2}\left(x^{1}\right)\right) \frac{\partial}{\partial x^{1}} R_{N} & -\frac{m^{2}}{L^{2}}\left(\omega^{2}-\Omega_{P N}^{2}\left(x^{1}\right)\right) R_{N} \\
& =q\left(x^{1}, \omega, m\right)
\end{aligned}
$$

Here $q\left(x^{1}, \omega, m\right)=\left\langle\tilde{q}_{m \omega} P_{N}\right\rangle$.

Let us consider the behavior of the wave vector radial component, $k_{1}$, determined in the WKB approximation where it is assumed that $R_{N} \propto \exp i \int k_{1} d x^{1}$. It can be shown that

$$
k_{1}^{2}\left(\omega, x^{1}\right)=\frac{m^{2}}{L^{2}} \frac{\Omega_{P N}^{2}\left(x^{1}\right)-\omega^{2}}{\omega^{2}-\Omega_{T N}^{2}\left(x^{1}\right)}
$$

(e.g., Klimushkin et al., 2004). It can be seen from this equation that the toroidal frequency $\Omega_{T N}$ corresponds to the mode resonance $\left(k_{1} \rightarrow \infty\right)$ while the poloidal frequency corresponds to the mode cut-off $\left(k_{1}=0\right)$.

Both the toroidal and the poloidal frequencies can be plotted as functions of the radial coordinate. The equation $k_{x}\left(\omega, x^{1}\right)=0$ at fixed $\omega$ has a solution $x_{P N}^{1}$ which can be found graphically as the intersection point of the function $\Omega_{P N}\left(x^{1}\right)$ curve and the horizontal line for the frequency $\omega$ (see Fig. 2). The magnetic shell with a radial coordinate $x_{P N}^{1}$ will be referred to as the poloidal surface. Similarly, a toroidal (or resonance) surface with a radial coordinate $x_{T N}^{1}$ can be introduced as a solution of the equation $k_{x}\left(\omega, x^{1}\right)=$ $\infty$ for fixed $\omega$. If $\Omega_{P}\left(x^{1}\right)>\Omega_{T}\left(x^{1}\right)$ and both functions are decreasing with $x^{1}$, then $x_{P}(\omega)>x_{T}(\omega)$. As can

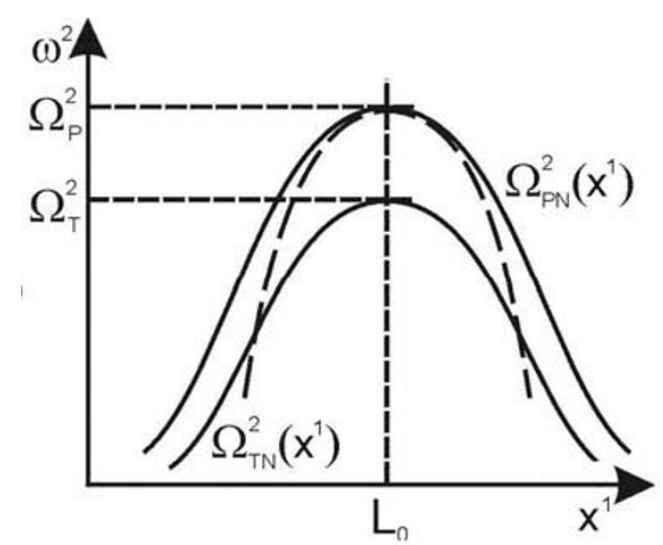

Fig. 3. Dependence of the toroidal $\Omega_{T N}$ and poloidal $\Omega_{P N}$ frequencies on the radial coordinate $x^{1}$ in the region of the transverse resonator on the plasmapause.

be seen from Eq. (11) and Fig. 2, the transparent region (where the mode is localized), where $k_{1}^{2}>0$, generally lies between the poloidal and the toroidal surfaces (Leonovich and Mazur, 1993; Mager and Klimushkin, 2002).

\section{Transverse Resonator on the Plasmapause}

If the poloidal frequency $\Omega_{P N}\left(x^{1}\right)$ has extremes as a function of the radial coordinate, there can exist a transverse resonator (or azimuthal waveguide), that is a transparent region bounded on both sides by cut-off surfaces. In this case the wave is channeled along the azimuth and trapped between the cut-off shells, being a standing wave in the direction transverse to the magnetic shells (Vetoulis and Chen, 1994; Leonovich and Mazur, 1995; Denton and Vetoulis, 1998; Klimushkin, 1998). As is evident from Fig. 2, one such region is located in the vicinity of the plasmapause and the other can be located in the vicinity of the ring current. The eigenfrequency of the resonator is determined from the condition that the number of half-waves between the cutoff surfaces should be an integer. The radial wave vector $k_{1}$ depending on the wave frequency $\omega$ leads to frequency quantization.

Whether the resonator is situated at the poloidal frequency minima or maxima depends on the sign of the difference between the toroidal and poloidal frequency $\Omega_{T N}-$ $\Omega_{P N}$ in this region. As can be seen from (11), if this difference is positive, the resonator is situated near the minimum of the function $\Omega_{P N}\left(x^{1}\right)$ (since the radial wave vector squared is positive there). Otherwise, it is situated near its maximum (Fig. 3).

If the finite plasma pressure is taken into account, the resonator at the minimum and maximum of the $\Omega_{P N}$ function is possible in the vicinity of the ring current and the outer edge of the plasmapause, respectively (Klimushkin et al., 2004). Let us consider the resonator on the outer edge plasmapause. For the sake of simplicity, we can employ the parabolic expansion

$$
\Omega_{P N}^{2}\left(x^{1}\right)=\Omega_{P}^{2}\left[1-\left(\frac{x^{1}-L_{0}}{l}\right)^{2}\right],
$$

where $\Omega_{P}$ and $L_{0}$ are the maximum value and position of 
$\Omega_{P N}\left(x^{1}\right)$, respectively (see Fig. 3 ). Further, we can neglect the toroidal frequency variation across the resonator:

$$
\Omega_{T N}\left(x^{1}\right)=\text { const }=\Omega_{T},
$$

where $\Omega_{T}=\Omega_{T N}\left(L_{0}\right)$ (see Fig. 3).

Equation (10) can be reduced to the form

$$
\frac{\partial^{2}}{\partial \xi^{2}} R+\left(\sigma-\xi^{2}\right) R=q \frac{L_{0} l}{m \Omega_{P}}\left(\omega^{2}-\Omega_{T}^{2}\right)^{-1 / 2},
$$

Here

$$
\xi=\left(\frac{m \Omega_{P}}{L_{0} l}\right)^{1 / 2}\left(\omega^{2}-\Omega_{T}^{2}\right)^{-1 / 4} x,
$$

where $x=x^{1}-L_{0}$,

$$
\sigma=\frac{m l}{L_{0} \Omega_{P}} \cdot \frac{\Omega_{P}^{2}-\omega^{2}}{\sqrt{\omega^{2}-\Omega_{T}^{2}}} .
$$

Let us ignore for now the right hand side of Eq. (14). The equation then is of the same form as is the Schrödinger equation for a harmonic oscillator. As is well known, it has a solution bounded outside of the region of mode localization. This requires that the parameter $\sigma$ be quantized, $\sigma=2 n+1$, where $n=0,1,2, \ldots$ is an integer. The $n$ value can be called the radial harmonic number. The wave frequency quantization condition is

$$
\omega_{n}^{2}(m)=\Omega_{P}^{2}-\Delta \omega_{n}^{2}(m),
$$

where

$$
\begin{gathered}
\Delta \omega_{n}^{2}(m)=-\frac{1}{2}\left(\frac{L_{0} \Omega_{P}}{m l}\right)^{2}(2 n+1)^{2}+ \\
+\frac{1}{2} \sqrt{\left(\frac{L_{0} \Omega_{P}}{m l}\right)^{4}(2 n+1)^{4}+4 \Delta \Omega^{2}\left(\frac{L_{0} \Omega_{P}}{m l}\right)^{2}(2 n+1)^{2}} \\
\Delta \Omega^{2}=\Omega_{P}^{2}-\Omega_{T}^{2} .
\end{gathered}
$$

It follows from Eq. (16) that the eigenfrequencies are limited by $\Omega_{T N}$ and $\Omega_{P N}$, and the difference between the eigenfrequencies increases with decreasing azimuthal wave number $m$ (Fig. 4). The eigenfrequencies characterize the resonator as a whole and do not depend on the radial coordinate.

The eigenfunctions are given by the expression

$$
R_{n}=\pi^{-1 / 4} 2^{-n / 2}(n !)^{-1 / 2} H_{n}(\xi) e^{-\xi^{2} / 2},
$$

where $H_{n}$ is the Hermite polynomial. The transversal structure of several resonator harmonics $R_{n}$ are shown in Fig. 5. Note that the radial structure of the principal radial harmonic, $n=0$, is described by the Gaussian function, $\Phi \propto$ $e^{-\xi^{2} / 2}$. In terms of the $\Phi$ function, all the harmonics peak near the center of the resonator. The radial and azimuthal components of the wave electric field are determined by the expressions $E_{1}=-\partial \Phi / \partial x^{1}$ and $E_{2}=-i k_{2} \Phi$, respectively. Hence, the azimuthal component of the wave electric field and, consequently, the radial component of the magnetic field follow the same pattern (17) as the $\Phi$ function and have maxima near the center of the resonator. The peak

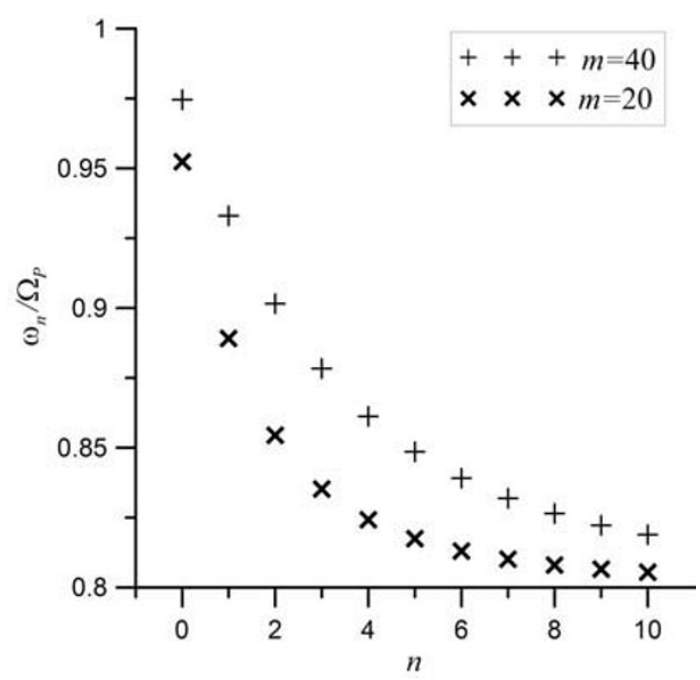

Fig. 4. Dependence of the resonator eigenfrequencies on harmonic number $n$ for different azimuthal wave numbers $m$. Here $\Omega_{T}=0.8 \Omega_{P}$.

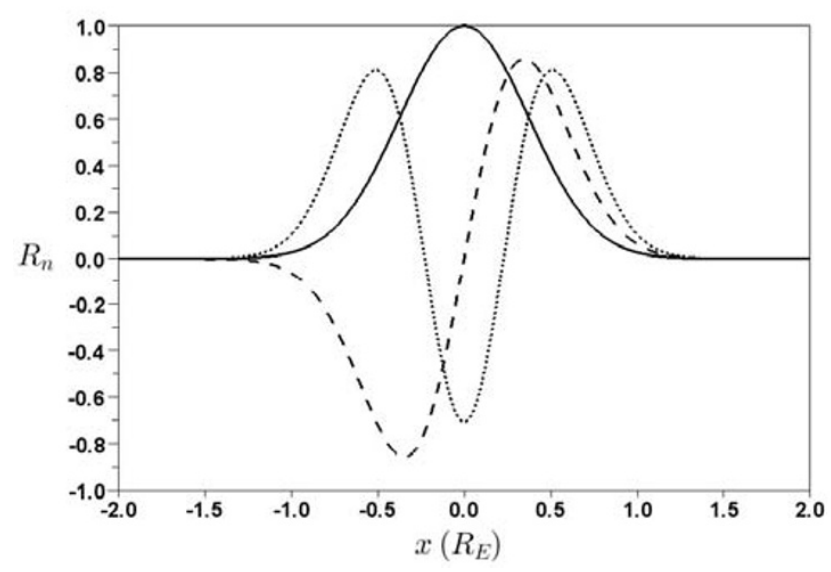

Fig. 5. Transversal structure of the first three resonator harmonics.

values of the radial component of the electric field and the azimuthal component of the magnetic field are concentrated at the locations with the largest derivatives $\partial E_{2} / \partial x^{1}$. Thus, the maximum of the poloidal component is located at the same position as the dip of the toroidal component.

\section{Wave Field Structure in a Resonator Excited by a Nonstationary Source}

Now let us return the source term into Eq. (14). The wave field in the resonator then is the superposition of all excited harmonics. Inhomogeneous Eq. (14) satisfying the boundary condition (5) can be solved using the above solution to the homogeneous equation:

$$
R_{N}(x, \omega, m)=\sum_{n=0}^{\infty} \frac{C_{n}}{\left(\sigma-\sigma_{n}\right)\left(\omega^{2}-\Omega_{T}^{2}\right)^{1 / 2}} R_{n}
$$

or

$$
R_{N}(x, \omega, m)=\frac{L_{0} \Omega_{P}}{m l} \sum_{n=0}^{\infty} \frac{C_{n}}{r_{n}(\omega)} R_{n} .
$$


Here

$$
\begin{gathered}
r_{n}(\omega)=\Omega_{P}^{2}-\omega^{2}-\left(\Omega_{P}^{2}-\omega_{n}^{2}\right) \sqrt{\frac{\omega^{2}-\Omega_{T}^{2}}{\omega_{n}^{2}-\Omega_{T}^{2}}} . \\
C_{n}=\frac{L_{0} l}{m \Omega_{P}} \int_{-\infty}^{+\infty} q R_{n} d \xi .
\end{gathered}
$$

The superposition of many transverse harmonics with different eigenfrequencies must result in oscillation amplitude modulation.

In what follows, we will deal with the spatio-temporal structure of the resonator modes excited by a non-stationary external azimuthal current. The source term $Q$ can then be represented as

$$
Q\left(x^{1}, x^{2}, t\right)=-\frac{4 \pi}{c^{2}} \sqrt{g} \frac{\partial}{\partial x^{2}} \frac{\partial}{\partial t} j_{\mathrm{ext}}^{2},
$$

where $j_{\text {ext }}^{2}=j_{\text {ext }} / \sqrt{g_{2}}$ is the contravariant azimuthal projection of the vector $\vec{j}_{\text {ext }}$. The wave equation (2) with a nonzero right-hand side can be solved via a reverse Fourier transform of the solution (7):

$$
\Phi\left(x^{1}, x^{2}, x^{3}, t\right)=\int_{-\infty}^{+\infty} d \omega \int_{-\infty}^{+\infty} d m \Phi_{m \omega} e^{i m \varphi-i \omega t} .
$$

Thus, according to Eqs. (22), (8), the solution of the wave equation (2) is

$$
\Phi\left(x^{1}, x^{2}, x^{3}, t\right)=\mathcal{R}_{N}\left(x^{1}, x^{2}, t\right) P_{N}\left(x^{1}, x^{3}\right),
$$

where the function

$$
\mathcal{R}_{N}\left(x^{1}, x^{2}, t\right)=\int_{-\infty}^{+\infty} d \omega \int_{-\infty}^{+\infty} d m R_{N}(m, \omega) e^{i m \varphi-i \omega t}
$$

defines both the transverse structure and evolution of the wave.

Let us further examine two different kinds of nonstationary sources: the impulsive source and the moving source comprised of drifting substorm-injected particles.

\subsection{Impulse source}

In the case of impulse excitation, the source current is given by the expression

$$
j_{\mathrm{ext}}^{2}=j_{0}(\varphi) \delta(t)
$$

Then in Eq. (10)

$$
\begin{gathered}
q\left(x^{1}, \omega, m\right)=q_{0} m \omega \\
q_{0}=-\frac{1}{\pi c^{2}} \int_{-\infty}^{+\infty} e^{-i m \varphi}\left\langle j_{0} \sqrt{g} T_{N}\right\rangle d \varphi
\end{gathered}
$$

and in Eq. (18)

$$
C_{n}=\frac{L_{0} l}{\Omega_{P}} q_{0} \omega \tilde{C}_{n}
$$
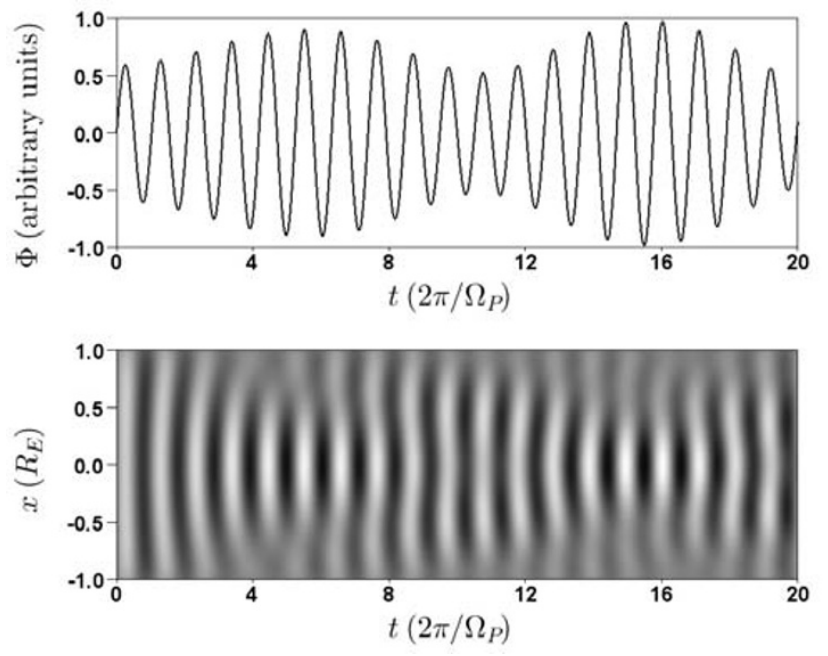

Fig. 6. Total wave field for an impulse source as a function of time at the center of the resonator (top panel) and as a function of distance from the center and time (bottom panel).

where

$$
\tilde{C}_{n}=\left\{\begin{array}{l}
0, \text { when } n=2 j+1,(j=0,1 \ldots), \\
\pi^{-1 / 4} 2^{-j} \sqrt{(2 j) !}(j !)^{-1} \sqrt{2 \pi}, \text { when } n=2 j .
\end{array}\right.
$$

Integrating over $\omega$, taking into account the two poles $\pm \omega_{n}$ of the integrated function, we finally obtain from Eqs. (24) and (18) the solution for a single Fourier $m$-harmonic

$$
\mathcal{R}_{N}=2 \pi i q_{0} \frac{L_{0}^{2}}{m} \sum_{n=0}^{\infty} \tilde{C}_{n} \tilde{R}_{n}(x) \cos \left(\omega_{n} t\right) e^{i m \varphi} .
$$

Here

$$
\tilde{R}_{n}(x)=\frac{R_{n}\left(\xi_{n}\right)}{1+\frac{1}{2} \frac{\Omega_{P}^{2}-\omega_{n}^{2}}{\omega_{n}^{2}-\Omega_{T}^{2}}},
$$

where

$$
\xi_{n}=\left(\frac{m \Omega_{P}}{L_{0} l}\right)^{1 / 2}\left(\omega_{n}^{2}-\Omega_{T}^{2}\right)^{-1 / 4} x
$$

Figure 6 shows the evolution of the impulse excited wave computed for the azimuthal number $m=20$ and $20 \%$ difference between the poloidal $\Omega_{P}$ and toroidal eigenfrequency $\Omega_{T}$. As expected, the superposition of different transvere harmonics results in amplitude modulation.

As seen in Fig. 7 the wave amplitude strongly depends on azimuthal wave number $m$. The amplitude rapidly increases from $m=0$ to $m \sim 10$ then more gradually decreases with increasing $m$. Thus, the oscillations with moderately high $m$ numbers will dominate in the total wave field.

\subsection{Moving source}

Another kind of non-stationary source is a cloud of substorm-injected energetic particles gradient-curvature drifting in the azimuthal direction. Let us assume that such a source acquires a field-aligned structure just after the injection, which requires the condition $\omega \ll \omega_{b}$ to be satisfied, where $\omega_{b}$ is the bounce frequency. The contravariant azimuthal projection of the external current is then

$$
j_{\mathrm{ext}}^{2}=e n_{0} \omega_{d} \delta\left(\varphi-\omega_{d} t\right) \Theta(t)
$$




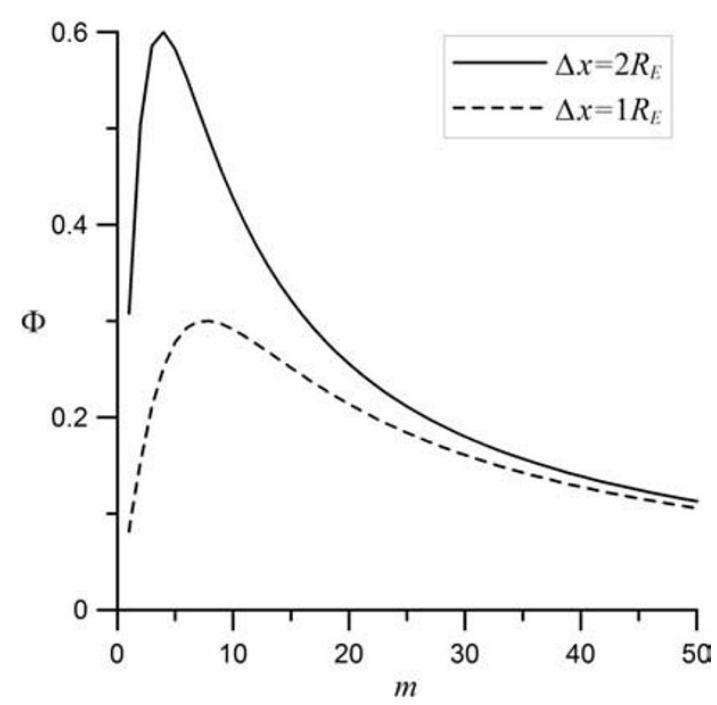

Fig. 7. Dependence of $n=0$ resonator harmonic amplitude on azimuthal wave number $m$ for different resonator widths $\Delta x$.

where $\omega_{d}\left(x^{1}\right)$ is the bounce-averaged angular drift velocity, $e$ and $n_{0}$ are the electric charge and number density of the particles, $\varphi$ is the azimuthal angle, which can be used as the $x^{2}$ coordinate. The physical component of the current can be obtained using the linear drift velocity $V=\sqrt{g_{2}} \omega_{d}$ instead of the angular velocity in Eq. (29). Thus, for this kind of source in Eq. (10)

$$
\begin{gathered}
q\left(x^{1}, \omega, m\right)=q_{0} m \omega \delta\left(\omega-m \omega_{d}\right), \\
q_{0}=-2 \frac{e \omega_{d}}{c^{2}}\left\langle n_{0} \sqrt{g} T_{N}\right\rangle
\end{gathered}
$$

(Mager and Klimushkin, 2007, 2008).

Consider the case when $\omega_{d}$ is independent of $x^{1}$. Then

$$
C_{n}=\frac{L_{0} l}{m \Omega_{P}} q_{0} m \omega \delta\left(\omega-m \omega_{d}\right) \tilde{C}_{n},
$$

where

$$
\tilde{C}_{n}=\left\{\begin{array}{l}
0, \text { when } n=2 j+1,(j=0,1 \ldots), \\
\pi^{-1 / 4} 2^{-j} \sqrt{(2 j) !}(j !)^{-1} \sqrt{2 \pi}, \text { when } n=2 j .
\end{array}\right.
$$

As a result, the expression defining both the transverse structure and evolution of the wave field is

$$
\begin{aligned}
& \mathcal{R}_{N}(x, \varphi, t)=q_{0} L_{0}^{2} \sum_{n=0}^{\infty} \tilde{C}_{n} \\
& \times \int_{-\infty}^{+\infty} d \omega \int_{-\infty}^{+\infty} d m \frac{\omega R_{n} \delta\left(\omega-m \omega_{d}\right) e^{i m \varphi-i \omega t}}{m r_{n}(\omega)} .
\end{aligned}
$$

First we integrate over $\omega$ in Eq. (31). Since we have the delta function depending on $\omega$ under the integral, we obtain

$$
\begin{gathered}
\mathcal{R}_{N}(x, \varphi, t)=q_{0} L_{0}^{2} \omega_{d} \sum_{n=0}^{\infty} \tilde{C}_{n} \\
\times \int_{-\infty}^{+\infty} d m \frac{R_{n} e^{i m \varphi-i m \omega_{d} t}}{r_{n}\left(m \omega_{d}\right)}
\end{gathered}
$$

In order to integrate this we use the residue theorem. The poles of the function to be integrated (see definition of function $r_{n}(\omega)$, Eq. (19)) are given by the expression

$$
m^{2}=\frac{\omega_{n}^{2}(m)}{\omega_{d}^{2}}
$$

Solutions to this equation are limited, since $\omega_{n}$ is limited, $\Omega_{P}>\omega_{n}>\Omega_{T}$. Furthermore, the difference between the toroidal $\Omega_{T}$ and poloidal eigenfrequency $\Omega_{P}$ is usually small in the magnetosphere, $\Delta \Omega^{2}=\Omega_{P}^{2}-\Omega_{T}^{2} \ll \Omega_{T}^{2}, \Omega_{P}^{2}$. Given these facts, we can solve Eq. (33) approximately: let

$$
m^{2}=\frac{\Omega_{P}^{2}}{\omega_{d}^{2}}-\delta m^{2}
$$

where $\delta m^{2} \ll \Omega_{P}^{2} / \omega_{d}^{2}$, then we have from (33) and (16)

$$
\delta m^{2} \approx \frac{\Delta \omega_{n}^{2}\left(\Omega_{P} / \omega_{d}\right)}{\omega_{d}^{2}} .
$$

Thus we have two poles $\pm m_{n}$, where

$$
m_{n} \equiv \frac{\tilde{\omega}_{n}}{\omega_{d}}
$$

and $\tilde{\omega}_{n}=\omega_{n}\left(\Omega_{P} / \omega_{d}\right)$. Finally, we obtain an approximate expression for $\mathcal{R}_{N}$ :

$$
\begin{aligned}
& \mathcal{R}_{N} \approx 2 \pi q_{0} L_{0}^{2} \Theta\left(\omega_{d} t-\varphi\right) \\
& \quad \times \sum_{n=0}^{\infty} \frac{\tilde{C}_{n}}{\tilde{\omega}_{n}} \tilde{R}_{n}(x) \sin \left(\tilde{\omega}_{n} t-m_{n} \varphi\right) .
\end{aligned}
$$

Expression $\Theta\left(\omega_{d} t-\varphi\right)$ appears in Eq. (34) because the causality principle is taken into account. Here

$$
\tilde{\omega}_{n}^{2}=\Omega_{P}^{2}-\Delta \tilde{\omega}_{n}^{2}
$$

where

$$
\begin{gathered}
\Delta \tilde{\omega}_{n}^{2}=\frac{1}{2} \omega_{d}^{2}\left(L_{0} / l\right)^{2}(2 n+1)^{2} \\
\times\left[\sqrt{1+4\left(\Delta \Omega^{2} / \omega_{d}^{2}\right)\left(l / L_{0}\right)^{2} /(2 n+1)^{2}}-1\right],
\end{gathered}
$$

and

$$
\tilde{R}_{n}(x)=\frac{R_{n}\left(\xi_{n}\right)}{1+\frac{1}{2} \frac{\Omega_{P}^{2}-\tilde{\omega}_{n}^{2}}{\tilde{\omega}_{n}^{2}-\Omega_{T}^{2}}}
$$

where

$$
\xi_{n}=\left(\frac{m_{n} \Omega_{P}}{L_{0} l}\right)^{1 / 2}\left(\tilde{\omega}_{n}^{2}-\Omega_{T}^{2}\right)^{-1 / 4} x .
$$

Thus, a wave excited by a moving source has a welldefined azimuthal wave number, given by the wave frequency to source angular velocity ratio, $m \sim \Omega_{P} / \omega_{d}$. As can be seen in Fig. 8, the wave has the azimuthal (poloidal) component $E_{2}$ only of the wave electric field, at the center of the resonator, while the radial (toroidal) component $E_{1}$ becomes significant closer to the edge. The wave evolution at a fixed azimuthal location is the same as in the impulse case (see Fig. 6). Similar to the impulse source, the superposition of different transverse harmonics with different eigenfrequencies results in amplitude modulation. 


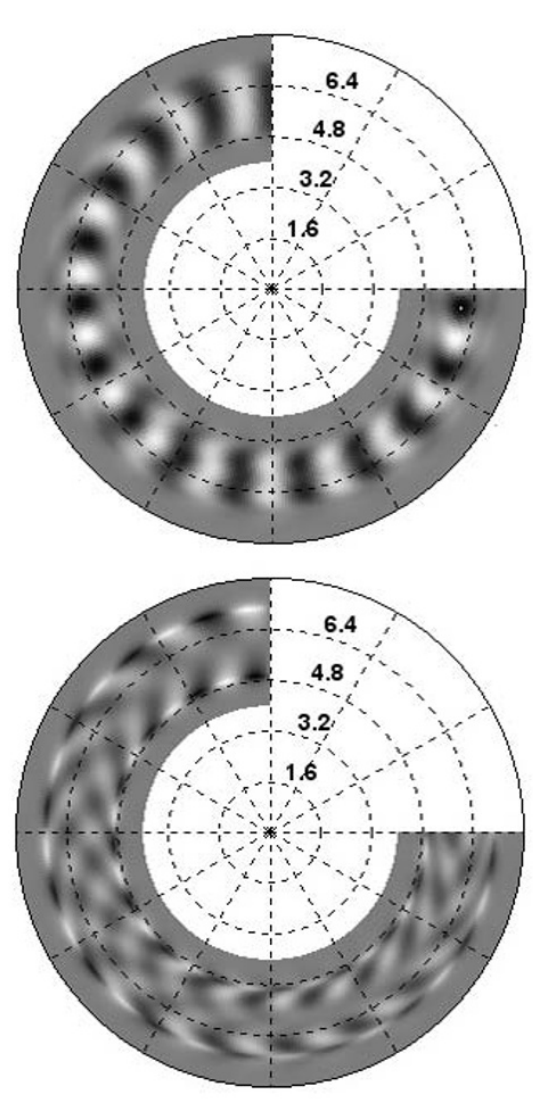

Fig. 8. Total wave field for an impulse source as a function of distance from the Earth center (in Earth radii) and azimuthal angle $\varphi$ : the azimuthal $E_{2}$ (top panel) and radial component $E_{1}$ (bottom panel) of the wave electric field.

\section{The Wave Structure in the Direction of the Ambient Magnetic Field}

When $\left|E_{2}\right| \gg\left|E_{1}\right|$ the parallel wave structure is determined by the poloidal operator. This condition is satisfied throughout the entire resonator except near the edges. The equation that determines the field-aligned structure of the poloidal mode can then be written as

$$
\hat{L}_{P}(\omega) \Phi=0 .
$$

Let us replace the field-aligned variable with $\xi$, determined as

$$
d \xi=\frac{\sqrt{g}}{g_{1}} d x^{3} .
$$

As a result, Eq. (35) can be represented in the form of the Schrödinger equation:

$$
\frac{\partial^{2}}{\partial \xi^{2}} \Phi+\frac{g_{1}}{g_{2}} \frac{\omega^{2}-H(\xi)}{A^{2}} \Phi=0
$$

where the term

$$
H(\xi)=-A^{2} \eta=A^{2} 2 K\left(\frac{4 \pi}{c} \frac{J}{B}+K \gamma \beta\right)
$$

is often called the ballooning term.

For heuristic purposes, Eq. (36) can be solved in the WKB approximation. The resulting field-aligned component of the wave vector is determined from the expression

$$
k_{\|}^{2}(\xi)=\frac{\omega^{2}-H(\xi)}{A^{2}} .
$$
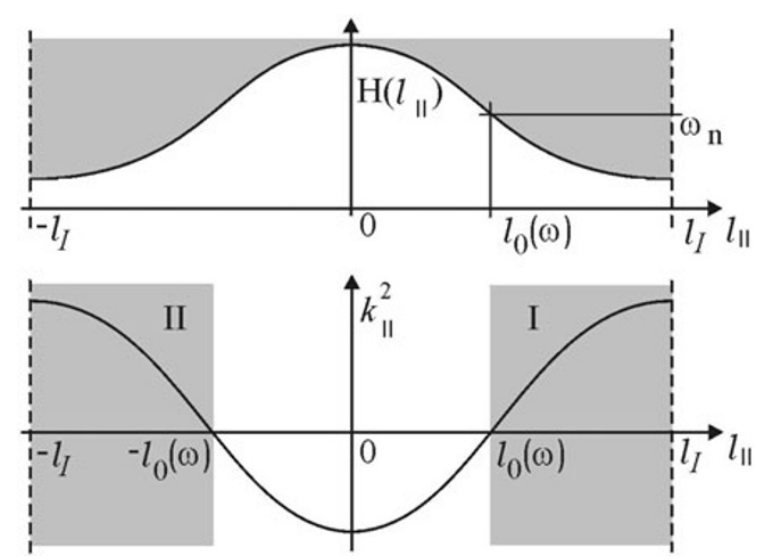

Fig. 9. Sketch demonstrating the dependence of $H$ (top) and $k_{\|}^{2}$ (bottom) on $l_{\|}$and showing the probable locations of the transparent and opaque regions. Here $\pm l_{0}$ are the turning points for a harmonic with eigenfrequency $\omega_{n}, \pm l_{I}$ are the field-line intersection points with the ionosphere. The shaded areas, I and II, correspond to the transparent regions.

In a cold plasma, when $H=0$, an Alfvén wave has no turning point, that is $k_{\|}^{2}>0$ everywhere. Thus the entire field line between the conjugate ionospheres is transparent for the shear Alfvén wave and the oscillation has a familiar sinusoidal structure.

In a finite-pressure plasma, an interesting effect occurs when the pressure gradient is outward: $\partial P / \partial x^{1}>0$. Let us find a region along the field line where the function $H(\xi)$ has a maximum. Usually, the second term in (37) dominates, thus $H$ varies along the parallel coordinate as $H \propto K^{2} \rho^{-1}$, where $\rho$ is the plasma density. The plasma pressure is to be constant along the field line, whereas the field line curvature $K$ and inverse density $\rho^{-1}$ both peak near the equator. Therefore, $H>0$ and the function $H(\xi)$ has a maximum at the equator (Fig. 9).

As follows from Eq. (38), the function $k_{\|}^{2}(\xi)$ in this case can reverse its sign along the field line. The point $l_{0}$, where $k_{\|}^{2}\left(l_{0}\right)=0$, is the turning point for a poloidal Alfvén wave. The region where $k_{\|}^{2}<0$, is an opaque region, where the wave becomes evanescent, and the region where $k_{\|}^{2}>0$ is transparent for waves.

When $H$ has a maximum at the magnetospheric equator, which is usually the case, the opaque region is located in the vicinity of the magnetospheric equator, where the field line curvature reaches its highest value (Mager et al., 2009b; Mazur et al., 2012). Thus, two sub-resonators (regions I and II in Fig. 9) form bounded by the ionosphere and the turning point near the equator, $\pm l_{0}$. However, due to the tunneling effect part of the wave energy leak out the ionospheric sub-resonators and forms wave field with small, but finite amplitude even in the opaque region. If this region is wide enough, this amplitude is exponentially small in its center and there is practically no coupling between the subresonators in the Northern and Southern hemispheres. Otherwise, the oscillations are coupled and the total wave functions $\Phi_{N}$ are composed of symmetric and antisymmetric combinations of the harmonics in regions I and II. The coupling of these two sub-resonators influences also the wave eigenfrequency. 

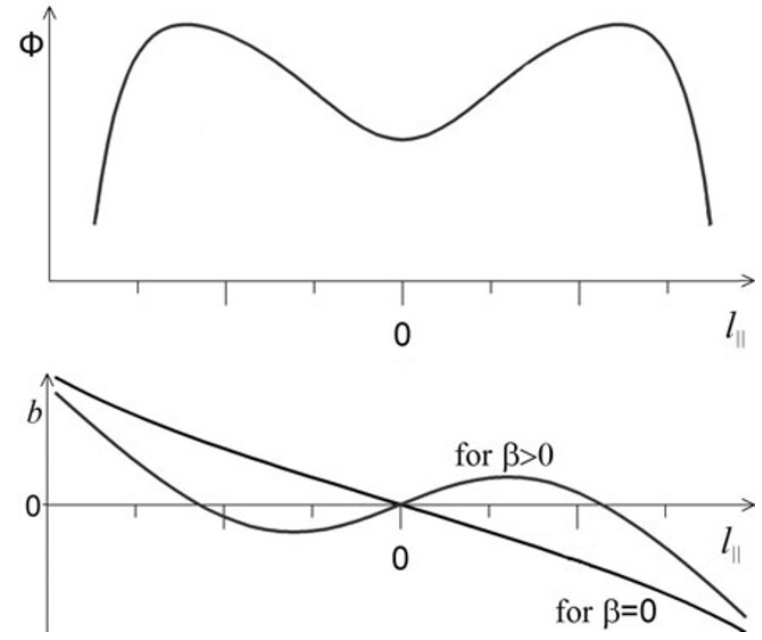

Fig. 10. Field-aligned structure of the fundamental harmonic. Shown are the wave potential $\Phi(\beta>0$ case) and magnetic field $b(\beta=0$ and $\beta>0$ cases).

The structure of the wave potential $\Phi$ must be most deformed for the fundamental mode, in comparison to the cold plasma case, since the opaque region is largest and "deepest" for it. In a cold plasma, the amplitude of the electric field of the fundamental harmonic $(N=1)$ has a maximum at the equator. In a finite pressure plasma with an opaque region near the field-line top, it has a minimum at the equator and two maxima in both hemispheres, a total of three extremes (Fig. 10). The radial component of the wave magnetic field is proportional to the field-aligned derivative of potential azimuthal component of the wave's electric field. Hence, the magnetic field of the fundamental harmonic must have three nodes rather than one node as is the case in a cold plasma. The magnetic field of the second harmonic must have two nodes only, just like the $\beta=0$ case. For more detail see Mager et al. (2009b).

\section{Discussion}

The suggestion that the giant pulsations are oscillations trapped within a resonator on the outer edge of the plasmapause is a plausible explanation given the narrow radial localization of these waves. Since the resonator is bounded across the L-shells by two turning points and the vector of the Alfvén wave's electric field is proportional to the transverse wave vector, the wave must be poloidally polarized, especially near the center of the resonator. The maximum of the poloidal component should be located at the same position in the resonator as the dip of the toroidal component. The eigenfrequencies of the resonator are determined by its global properties (height, width, difference between the poloidal and toroidal frequencies inside the resonator), providing a possible explanation for the Pgs frequency being independent of the radial coordinate.

The resonator on the outer edge of the plasmapause owes its existence to the fact that the poloidal eigenfrequency is very sensitive to plasma pressure. An additional hint at the role of the pressure is the considerable compressional component of the wave magnetic field. When geomagnetic activity decreases, the plasmapause shifts away from the
Earth, into higher- $\beta$ regions (O'Brien and Moldwin, 2003), which offers an explanation for the occurrence of the giant pulsations in geomagnetically quiet periods.

The principal transverse harmonic of the resonator must be close to the Gaussian function, which offers a satisfactory explanation of the radial shape of the Pgs wave field. However, if the resonator is excited by some external source, its structure must be the superposition of all transverse harmonics. In order to explain the amplitude modulation it is sufficient to suppose that the resonator is excited by some non-stationary process. Both the non-stationary sources considered lead to similar temporal structures of the oscillation shown in Figs. 6 and 8. Such structure was observed earlier with radars for a moderately high- $m$ pulsation (Wright and Yeoman, 1999), and was interpreted in terms of transverse resonator theory (Yeoman et al., 2012).

The assumption on the resonator can explain also absence of the transformation from the poloidally to toroidally polarized wave expected from the theory of the phase mixing of the Alfvén wave with the continuous spectrum (Chisham et al., 1997). As is seen from Figs. 6 and 8, there is no such transformation of the discrete resonator modes. Thus, we may conclude that the presence of a transverse resonator can at least explain the basic properties of the giant pulsations.

However, some peculiarities of Pgs cannot be directly explained in this model. Among them are localization to the dawn sector, occurrence at the equinox and at the solar minimum (Brekke et al., 1987). To include these features in the general picture, it would be necessary to advance the theory, that is, to find the dependence of the resonator properties on the geomagnetic indexes and to generalize it for the case of the azimuthally-inhomogeneous magnetosphere.

Then, the generation mechanism remains to be unclear. The moving source concept in this paper assumes the wave electric field to be symmetric with respect to the equator. This concept is in agreement with the observations, outlined in the Introduction, that Pgs represent an odd parallel harmonic. It also assumes, however, the resonance condition

$$
\omega-m \omega_{d}=0
$$

to hold, which requires, for $|m| \sim 15-20$, very large energies for the particles. Besides, there is no simple way to distinguish such a moving source from the gradient instability, which assumes the same resonance condition.

The alternative could be the bump-on-tail instability, which agrees with the resonance condition

$$
\omega-m \omega_{d}-K \omega_{b}=0,
$$

where $\omega_{b}$ is the bounce frequency and $K \neq 0$ is the bounce harmonic number. Note that the resonance is possible only for even $K$, since $\mathrm{Pgs}$ are usually the fundamental parallel harmonic. In this case, 10-20 keV particles can generate waves with very high $m$ numbers (Mager and Klimushkin, 2005). For moderately high $m$ Pgs and for such energies the middle term of the resonance condition becomes insufficient, and is in effect reduced to $\omega-K \omega_{b} \approx 0$ (Chisham et al., 1992). This then raises the question of how to determine the azimuthal wave number and the wave propagation 
direction. Moreover, wave generation should be considered as an impulsive process, but the instability theory has only been developed for the stationary wave field structures. As for the moving source theory, it has to date been developed for the $\omega \ll \omega_{b}$ case only.

Finally, the close vicinity of Pg location to the auroral regions raises questions of the role of the ionosphere in $\mathrm{Pg}$ generation by means of, e.g., feedback instability (e.g., Watanabe, 2010) or changing ionospheric conductivity (Maltsev et al., 1974), although both the mechanisms are suggested as explanations of a quite different kind of ULF waves, the irregular pulsations Pi2. The field aligned currents in the auroral regions further complicate the mode structure by, e.g., influencing the poloidal eigenfrequency (Klimushkin and Mager, 2004b). The impact of this factor is unclear.

\section{Conclusions}

The paper assumes that giant pulsations are oscillations trapped within a resonator appearing, due to finite plasma pressure, on the outer edge of the plasmapause. The general features of this resonator that explain the basic properties of the giant pulsations.

1) The wave in the resonator is bounded across the Lshells by two turning points. The giant pulsations also have narrow radial localizations and are usually observed on the outer edge of the plasmapause where such a transversal resonator can exist.

2) The wave is predominantly poloidally polarized. At the center of the resonator, it has the poloidal component of the wave field only, while the toroidal component becomes significant closer to its edge. A similar behavior of polarization across the L-shells is observed for the giant pulsations.

3) In regions where toroidal and poloidal eigenfrequency profiles are monotonic, oscillation frequencies depend on the L-shells. Conversely, the resonator eigenfrequencies are determined by its global properties. This is a possible explanation for the Pgs frequency being independent of the radial coordinate. Constant wave frequencies over the Lshells mean that the resonator modes are not subject of the phase mixing phenomenon which explains absence of the transformation from the poloidally to toroidally polarized for the Pg waves.

4) The wave in the resonator is subject to amplitude modulation due to superposition of harmonics with close eigenfrequencies. Such modulation is observed for the giant pulsations.

5) The resonator on the outer edge of the plasmapause can exist when $\beta$ is high enough and the poloidal eigenfrequency becomes higher than the toroidal eigenfrequency. The giant pulsations are usually observed in geomagnetically quiet periods when the plasmapause is shifted from the Earth into the higher- $\beta$ regions. Moreover, they have a considerable compressional component of the wave magnetic field, which also indicates significant plasma pressure.

6) As seen from observations, the giant pulsations often represent the fundamental $(N=1)$ harmonic of the standing wave. In this case, plasma pressure has a strong influence on the parallel structure of the poloidal wave. The magnetic field of the fundamental harmonic must have three nodes, rather than one as in the case of cold plasma. Such a structure has not yet been observed, possibly due to difficulties of spacecraft observations along magnetic field lines.

Acknowledgments. The work was supported by RFBR grants 12-05-00121-a and 12-05-98522-r-vostok-a, Program \#22 of the Presidium of the Russian Academy of Sciences.

\section{References}

Agapitov, A. V. and O. K. Cheremnykh, Polarization of ULF waves in the Earth's magnetosphere, Kinemat. Phys. Celest. Bodies, 27, 117-123, 2011.

Annexstad, J. O. and C. R. Wilson, Characteristics of Pg micropulsations at conjugate points, J. Geophys. Res., 73, 1805-1818, 1968.

Baddeley, L. J., T. K. Yeoman, and D. M. Wright, HF doppler sounder measurements of the ionospheric signatures of small scale ULF waves, Ann. Geophys., 23, 1807-1820, 2005.

Brekke, A., T. Feder, and S. Berger, Pc4 giant pulsations recorded in Tromsø, 1929-1985, J. Atmos. Terr. Phys., 49, 1027-1032, 1987.

Chisham, G., Giant pulsations: An explanation for their rarity and occurrence during geomagnetically quiet times, J. Geophys. Res., 101, 24,755-24,764, 1996.

Chisham, G. and D. Orr, Statistical studies of giant pulsations (Pgs): Harmonic mode, Planet. Space Sci., 39, 999-1006, 1991.

Chisham, G., D. Orr, and T. K. Yeoman, Observations of a giant pulsation across an extended array of ground magnetometers and on auroral radar, Planet. Space Sci., 40, 953-964, 1992.

Chisham, G., I. R. Mann, and D. Orr, A statistical study of giant pulsation latitudinal polarization and amplitude variation, J. Geophys. Res., 102, 9619-9630, 1997.

Cramm, R., K.-H. Glassmeier, C. Othmer, K.-H. Fornasson, H.-U. Auster, W. Baumjohan, and E. Georgescu, A case study of a radially polarized Pc 4 event observed by the Equator-S satellite, Ann. Geophys., 18, 411415, 2000.

Denton, R. E. and G. Vetoulis, Global poloidal mode, J. Geophys. Res., 103, 6729-6739, 1998.

Engebretson, M. J., D. L. Murr, K. N. Ericson, R. J. Strangeway, D. M. Klumpar, S. A. Fuselier, L. J. Zanetti, and T. A. Potemra, The spatial extent of radial magnetic pulsations events observed in the dayside near synchronous orbit, J. Geophys. Res., 97, 13,741-13,758, 1992.

Glassmeier, K.-H., Magnetometer array observations of a giant pulsation event, J. Geophys., 48, 127-138, 1980.

Glassmeier, K.-H., Reply to the comment by I. R. Mann and G. Chisham, Ann. Geophys., 18, 167-169, 2000.

Glassmeier, K.-H., S. Buchert, U. Motschmann, A. Korth, and A. Pedersen, Concerning the generation of geomagnetic giant pulsations by driftbounce resonance ring current instabilities, Ann. Geophys. 17, 338-350, 1999.

Green, C. A., Observations of Pg pulsations in the Northern auroral zone and at lower latitude conjugate regions, Planet. Space Sci., 27, 63-77, 1979.

Green, C. A., Giant pulsations in the plasmasphere, Planet. Space Sci., 33, 155-168, 1985.

Guglielmi, A. V. and N. A. Zolotukhina, Excitation of Alfvén oscillations of the magnetosphere by the asymmetric ring current, Issled. geomagn. aeron. i fiz. Solntsa, 50, 129-137, 1980 (in Russian).

Hughes, W. J., R. I. McPherron, J. N. Barfield, and B. H. Mauk, A compressional Pc4 pulsation observed by three satellites in geostationary orbit near local midnight, Planet. Space Sci., 27, 821-840, 1979.

Kato, Y. and T. Watanabe, A possible explanation of cause of giant pulsations, Sci. Rep. Tohoku Univ. Ser. 5, Geophys., 6, 95-104, 1955.

Kato, Y. and T. Watanabe, Further study on the cause of giant pulsation, Sci. Rep. Tohoku Univ. Ser. 5, Geophys., 8, 1-10, 1956.

Klimushkin, D. Yu., Spatial structure of small-scale azimuthal hydromagnetic waves in an axisymmetric magnetospheric plasma with finite pressure, Plasma Phys. Rep., 23, 858-871, 1997.

Klimushkin, D. Yu., Resonators for hydromagnetic waves in the magnetosphere, J. Geophys. Res., 103, 2369-2375, doi:10.1029/97JA02193, 1998.

Klimushkin, D. Yu., The propagation of high- $m$ Alfvén waves in the Earth's magnetosphere and their interaction with high-energy particles, J. Geophys. Res., 105, 23,303-23,310, 2000.

Klimushkin, D. Yu., How energetic particles construct and destroy poloidal high- $m$ Alfvén waves in the magnetosphere, Planet. Space Sci., 55, 722- 
730, doi:10.1016/j.pss.2005.11.006, 2007.

Klimushkin, D. Yu. and P. N. Mager, The spatio-temporal structure of impulse-generated azimuthally small-scale Alfvén waves interacting with high-energy charged particles in the magnetosphere, Ann. Geophys., 22, 1053-1060, 2004a.

Klimushkin, D. Yu. and P. N. Mager, The structure of low-frequency standing Alfvén waves in the box model of the magnetosphere with magnetic field shear, J. Plasma Phys., 70, 379-395, 2004b.

Klimushkin, D. Yu. and P. N. Mager, Spatial structure and stability of coupled Alfvén and drift compressional modes in non-uniform magnetosphere: Gyrokinetic treatment, Planet. Space Sci., 59, 1613-1620, doi:10.1016/j.pss.2011.07.010, 2011.

Klimushkin, D. Yu. and P. N. Mager, Coupled Alfvén and driftmirror modes in non-uniform space plasmas: A gyrokinetic treatment, Plasma Phys. Control. Fusion, 54, 015006 (10pp), doi:10.1088/07413335/54/1/015006, 2012

Klimushkin, D. Yu., P. N. Mager, and K.-H. Glassmeier, Toroidal and poloidal Alfvén waves with arbitrary azimuthal wave numbers in a finite pressure plasma in the Earth's magnetosphere, Ann. Geophys., 22, 267288, 2004.

Klimushkin, D. Yu., I. Yu. Podshibyakin, and J. B. Cao, Azimuthally small-scale Alfvén waves in magnetosphere excited by the source of finite duration, Earth Planets Space, 59, 951-959, 2007.

Klimushkin, D. Yu., P. N. Mager, and K.-H. Glassmeier, Spatio-temporal structure of Alfvén waves excited by a sudden impulse localized on an L-shell, Ann. Geophys., 30, 1099-1106, doi:10.5194/angeo-30-10992012, 2012.

Kokubun, S., K. N. Erickson, T. A. Fritz, and R. L. McPherron, Local time asymmetry of Pc 4-5 pulsations and associated particle modulations at synchronous orbit, J. Geophys. Res., 94, 6607-6625, doi:10.1029/JA094iA06p06607, 1989.

Kurazhkovskaya, N. A., B. I. Klain, B. V. Dovbnya, and O. D. Zotov, On the relation of giant pulsations $(\mathrm{Pg})$ to pulsations in the Pc1 band (the "pearl" series), Int. J. Geomagn. Aeron., 5, GI2001, doi:10.1029/2003GI000062, 2004.

Lehnert, B., Magneto-hydrodynamic waves in the ionosphere and their application to giant pulsations, Tellus, 8, 241-251, doi:10.1111/j.21533490.1956.tb01217.x, 1956.

Leonovich, A. S. and V. A. Mazur, A theory of transverse small-scale standing Alfvén waves in an axially symmetric magnetosphere, Planet. Space Sci., 41, 697-717, 1993.

Leonovich, A. S. and V. A. Mazur, Magnetospheric resonator for transverse-small-scale standing Alfvén waves, Planet. Space Sci., 43, 881-883, 1995.

Leonovich, A. S. and V. A. Mazur, Standing Alfvén waves in an axisymmetric magnetosphere excited by a non-stationary source, Ann. Geophys., 16, 914-920, 1998.

Mager, P. N. and D. Yu. Klimushkin, Theory of azimuthally small-scale Alfvén waves in an axisymmetric magnetosphere with small but finite plasma pressure, J. Geophys. Res., 107, 1356, doi:10.1029/2001JA009137, 2002.

Mager, P. N. and D. Yu. Klimushkin, Spatial localization and azimuthal wave numbers of Alfvén waves generated by drift-bounce resonance in the magnetosphere, Ann. Geophys., 23, 3775-3784, doi:10.5194/angeo23-3775-2005, 2005.

Mager, P. N. and D. Yu. Klimushkin, On impulse excitation of the global poloidal modes in the magnetosphere, Ann. Geophys., 24, 2429-2433, 2006.

Mager, P. N. and D. Yu. Klimushkin, Generation of Alfvén waves by a plasma inhomogeneity moving in the Earth's magnetosphere, Plasma Phys. Rep., 33, 391-398, doi:10.1134/S1063780X07050042, 2007.

Mager, P. N. and D. Yu. Klimushkin, Alfvén ship waves: high- $m$ ULF pulsations in the magnetosphere generated by a moving plasma inhomogeneity, Ann. Geophys., 26, 1653-1663, 2008.

Mager, P. N., D. Yu. Klimushkin, and N. Ivchenko, On the equatorward phase propagation of high- $m$ ULF pulsations observed by radars, $J$. Atmos. Sol.-Terr. Phys., 71, 1677-1680, doi:10.1016/j.jastp.2008.09.001, 2009a.

Mager, P. N., D. Yu. Klimushkin, V. A. Pilipenko, and S. Schäfer, Fieldaligned structure of poloidal Alfvén waves in a finite pressure plasma, Ann. Geophys., 27, 3875-3882, 2009b.

Maltsev, Yu. P., S. V. Leontyev, and W. B. Lyatsky, Pi-2 pulsations as a result of evolution of an Alfvén impulse originating in the ionosphere during a brightening of aurora, Planet. Space Sci., 22, 1519-1533, 1974.

Mann, I. R. and G. Chisham, Comment on "Concerning the generation of geomagnetic giant pulsations by drift-bounce resonance ring current instabilities" by K.-H. Glassmeier et al., Ann. Geophysicae, 17, 338-350, (1999), Ann. Geophys., 18, 161-166, doi:10.1007/s00585-000-0161-4, 2000.

Mann, I. R., A. W. Wright, and A. W. Hood, Multiple-timescales analysis of ideal poloidal Alfvén waves, J. Geophys. Res., 102, 2381-2390, 1997.

Mazur, N. G., E. N. Fedorov, and V. A. Pilipenko, Dispersion relation for the ballooning modes and condition for their stability in circumterrestrial plasma, Geomagn. Aeron., 52, 603-612, 2012.

O'Brien, T. P. and M. B. Moldwin, Empirical plasmapause models from magnetic indices, Geophys. Res. Lett., 30(4), 1152, doi:10.1029/2002GL016007, 2003.

Ol', A. N., Long period gigantic geomagnetic pulsations, Geomagn. Aeron., 3, 113-120, 1963 (in Russian).

Ozeke, L. G. and I. R. Mann, Modeling the properties of high $m$ Alfvén waves driven by the drift-bounce resonance mechanism, J. Geophys. Res., 106, 15,583-15,597, 2001.

Pokhotelov, O. A., V. A. Pilipenko, and E. Amata, Drift anisotropy instability of a finite- $\beta$ magnetospheric plasma, Planet. Space Sci., 33, 1229-1241, 1985

Pokhotelov, O. A., Y. G. Khabazin, I. R. Mann, D. K. Milling, R. K. Shukla, and L. Stenflo, Giant pulsations: A nonlinear phenomenon, $J$. Geophys. Res., 105(A5), 10,691-10,702, doi:10.1029/1999JA900506, 2000a.

Pokhotelov, O. A., M. A. Balikhin, H. S.-C. K. Alleyne, and O. G. Onishchenko, Mirror instability with finite electron temperature effects, $J$. Geophys. Res., 105, 2393-2401, doi:10.1029/1999JA900351, 2000b.

Poulter, E. M., W. Allan, E. Nielsen, and K.-H. Glassmeier, Stare radar observations of a Pg pulsation, J. Geophys. Res., 88, 5668-5676, doi:10.1029/JA088iA07p05668, 1983.

Rolf, B., Giant micropulsations at Abisko, Terr. Magn., 36, 9-14, 1931.

Rostoker, G., H.-L. Lam, and J. V. Olson, PC 4 giant pulsations in the morning sector, J. Geophys. Res., 84(A9), 515-5166, doi:10.1029/JA084iA09p05153, 1979.

Schäfer, S., K.-H. Glassmeier, P. T. I. Eriksson, P. N. Mager, V. Pierrard, K.-H. Fornasson, and L. G. Blomberg, Spatio-temporal structure of a poloidal Alfveń wave detected by Cluster adjacent to the dayside plasmapause, Ann. Geophys., 26, 1805-1817, 2008.

Singer, H. J., W. J. Hughes, and C. T. Russel, Standing hydromagnetic waves observed by ISEE 1 and 2: Radial extent and harmonic, J. Geophys. Res., 87, 3519-3527, 1982.

Southwood, D. J., A general approach to low-frequency instability in the ring current plasma, J. Geophys. Res., 81, 3340-3348, doi:10.1029/JA081i019p03340, 1976.

Takahashi, K. and B. J. Anderson, Distribution of ULF-energy $(f<80$ $\mathrm{mHz}$ ) in the inner magnetosphere: A statistical analysis of AMPTE CCE magnetic field data, J. Geophys. Res., 97, 10,751-10,769, 1992.

Takahashi, K., N. Sato, J. Warnecke, H. Luehr, H. E. Spence, and Y. Tonegawa, On the standing wave mode of giant pulsations, J. Geophys. Res., 97, 10,717-10,732, doi:10.1029/92JA00382, 1992.

Takahashi, K., K.-H. Glassmeier, V. Angelopoulos, J. Bonnell, Y Nishimura, H. J. Singer, and C. T. Russell, Multisatellite observations of a giant pulsation event, J. Geophys. Res., 116, A11223, doi:10.1029/2011JA016955, 2011.

Tamao, T., Transmission and coupling resonance of hydromagnetic disturbances in the non-uniform Earth's magnetosphere, Sci. Rep. Tohoku Univ. Ser. 5, Geophys., 17, 43-72, 1965.

Taylor, M. J., G. Chisham, and D. Orr, Pulsating auroral forms and their association with geomagnetic giant pulsations, Planet. Space Sci., 37, 1477-1484, 1989.

Thompson, S. M. and M. G. Kivelson, New evidence for the origin of giant pulsations, J. Geophys. Res., 106(A10), 21,237-21,253, 2001.

Vetoulis, G. and L. Chen, Global structures of Alfvén-ballooning modes in magnetospheric plasmas, Geophys. Res. Lett., 21, 2091-2094, doi:10.1029/94GL01703, 1994.

Watanabe, T.-H., Feedback instability in the magnetosphereionosphere coupling system: Revisited, Phys. Plasmas, 17, 022904, doi:10.1063/1.3304237, 2010 .

Wright, D. M. and T. K. Yeoman, CUTLASS observations of a high- $m$ ULF wave and its consequences for the DOPE HF Doppler sounder, Ann. Geophys., 17, 1493-1497, doi:10.1007/s00585-999-1493-3, 1999.

Wright, D. M., T. K. Yeoman, I. J. Rae, J. Storey, A. B. Stockton-Chalk, J. L. Roeder, and K. J. Trattner, Ground-based and polar spacecraft observations of a giant $(\mathrm{Pg})$ pulsation and its associated source mechanism, 
J. Geophys. Res., 106, 10,837-10,852, 2001.

Yeoman, T. K., D. Yu. Klimushkin, and P. N. Mager, Intermediate- $m$ ULF waves generated by substorm injection: A case study, Ann. Geophys., 28, 1499-1509, 2010.

Yeoman, T. K., M. James, P. N. Mager, and D. Yu. Klimushkin, SuperDARN observations of high- $m$ ULF waves with curved phase fronts and their interpretation in terms of transverse resonator theory, J. Geophys. Res., 117, A06231, doi:10.1029/2012JA017668, 2012.

Zolotukhina, N. A., On excitation of Alfvén waves in the magnetosphere by a moving source, Issled. geomagn. aeron. i fiz. Solntsa, 34, 20-23, 1974 (in Russian).

Zolotukhina, N. A., P. N. Mager, and D. Yu. Klimushkin, Pc5 waves generated by substorm injection: A case study, Ann. Geophys., 26, 2053 2059, 2008.

P. N. Mager (e-mail: p.mager@iszf.irk.ru) and D. Yu. Klimushkin 